\title{
La France et sa langue en Méditerranée orientale au début du $\mathrm{XX}^{\mathrm{e}}$ siècle
}

\section{Patrick Cabanel}

\section{(2) OpenEdition \\ Journals}

Édition électronique

URL : https://journals.openedition.org/dhfles/142

DOI : $10.4000 /$ dhfles. 142

ISSN : 2221-4038

Éditeur

Société Internationale pour l'Histoire du Français Langue Étrangère ou Seconde

Édition imprimée

Date de publication : 1 janvier 2007

Pagination : 17-32

ISSN : 0992-7654

Référence électronique

Patrick Cabanel, «La France et sa langue en Méditerranée orientale au début du XXe siècle », Documents pour l'histoire du français langue étrangère ou seconde [En ligne], 38/39 | 2007, mis en ligne le 16 décembre 2010, consulté le 27 mai 2021. URL : http://journals.openedition.org/dhfles/142 ; DOI : https://doi.org/10.4000/dhfles.142

Ce document a été généré automatiquement le 27 mai 2021.

(c) SIHFLES 


\title{
La France et sa langue en Méditerranée orientale au début du $\mathrm{XX}^{\mathrm{e}}$ siècle
}

\author{
Patrick Cabanel
}

1 L'histoire du rayonnement mondial de la langue française au début du $\mathrm{XX}^{\mathrm{e}}$ siècle est une des plus riches et des plus passionnantes qui soient, non pas parce que le français aurait joui de quelque supériorité intrinsèque, mais parce qu'il a bénéficié d'une conjonction étonnante de facteurs positifs, dont certains, au départ, n'étaient rien d'autre que des drames, et même des fautes dont la France s'est rendue coupable. Seule l'Allemagne, dont on connaît et l'exceptionnel destin européen de la langue, et la gravité des fautes qu'elle a commises sous le nazisme et qui se sont finalement retournées contre la nation et sa langue (l'extermination des juifs a largement été un assassinat de la langue allemande1 ${ }^{1}$ ), présente une histoire (presque) aussi époustouflante. Peut-on, du reste, parler d'une seule langue française, quand des têtes et des bouches si différentes la pensaient et la parlaient, quand les littératures et les panthéons n'étaient pas les mêmes (Voltaire adulé ici, détesté là...), quand trois " confessions » au moins, en comptant la laïque, lui fournissaient instituteurs, agents et écrivains? Il y avait le français catholique, distribué par les missionnaires et les congrégations religieuses enseignantes ou hospitalières; le français juif, répandu par les maîtres et maîtresses de l'Alliance israélite universelle fondée en 1860 ; et le français laïque, qu'il le soit au sens républicain officiel dans les territoires coloniaux, protectorats compris, ou au sens militant et proprement missionnaire de la Mission laïque française (1902). À quoi l'historien doit ajouter, même si l'espace méditerranéen n’a pas été concerné par cette espèce, le français huguenot, celui que les quelque 250000 protestants partis de France au moment ou à la suite de la révocation de l'édit de Nantes, en 1685, n'ont pas manqué de répandre dans l'Europe du nord-ouest.

Voilà beaucoup de milieux, de réseaux, d'accents et de lexiques pour une seule langue. Voilà un temps long de son exportation, puisque les protestants ont commencé à fuir la France dès les années 1560 et ont gardé la langue au Refuge, au moins pour les 
exercices religieux, jusqu'au début du $\mathrm{XIX}^{\mathrm{e}}$ siècle en Allemagne, jusqu'à nos jours en Hollande. Voilà, dans les années 1880-1940, un extraordinaire enchevêtrement de paroles françaises dans le monde méditerranéen, puisque catholiques, laïques, juifs, coloniaux les prononcent également. Et leurs élèves, dont le plus grand - s'il faut en citer un seul, ce sera celui-là -, débarqué enfant à Marseille avec ses parents de Corfou, se lit aujourd'hui en Pléiade: on aura reconnu Albert Cohen, très grand écrivain français, très grand écrivain juif méditerranéen. Sur ce sujet, qui appellerait de vastes développements et que j'ai pour ma part abordé dans de précédentes publications ${ }^{2}$, je ne retiendrai que deux points: le paradoxe de la faute et de l'exil, qui se muent en féconde dissémination; et l'extraordinaire accumulation d'atouts qui va rendre à peu près irrésistible la conquête d'une partie du monde par le français, même si surgit, déjà, une inquiétude pour l'avenir, peut-être aussi française que le sentiment d'une élection face à l'humanité...

\section{La faute de la nation et la dissémination de sa langue}

3 À trois reprises au moins, et pour ne rien dire d'épisodes intermédiaires ${ }^{3}$, la France a contraint à l'exil, même s'il ne s'agit pas d'expulsion ${ }^{4}$, des groupes compacts d'hommes et de femmes appartenant souvent à ses élites : les protestants sous la monarchie très chrétienne, spécialement à la fin du XVII ${ }^{e}$ siècle ; les " émigrés ", nobles et prêtres, sous la Révolution; enfin, les congréganistes frappés par les deux grandes lois anticléricales de juillet 1901 et juillet 1904. On peut apprécier de façons très diverses ces exils religieux ou politiques (ou l'un et l'autre) et l'attitude des régimes qui les ont rendus nécessaires : dans quel camp la justice se trouvait-elle ? C'est à chacun de se prononcer. Très vite, pour donner un seul exemple, un grand ingénieur et serviteur de Louis XIV, Vauban, avait plaidé pour le retour des huguenots, et une littérature qui ne compte pas que des auteurs protestants a regretté la double faute commise en 1685 contre l'humanité, dirions-nous, mais aussi contre la France, privée d'une partie importante de ses soldats, ingénieurs, techniciens, commerçants, industriels, intellectuels ${ }^{5} . .$. Mais, laissant là tout souci de justice et de morale et même de patriotisme étroit, l'historien de la langue, lui, est en droit de se réjouir ; en quoi, du reste, il ne manque pas non plus de patriotisme, dès lors que la nation est aussi une langue et que l'empire linguistique, pour être immatériel, n'est pas une moindre province du rayonnement international d'un pays.

Devenus ses meilleurs agents, en effet, des dizaines de milliers d'exclus ou d'exilés ont emporté et diffusé la langue française. Les exils huguenot et surtout congréganiste sont constitués pour une forte part de professionnels de la culture, du livre et de l'école : la presque totalité des écrivains, théologiens, pasteurs, professeurs d'académies protestantes, autour de 1685, des milliers d'enseignants congréganistes de tous niveaux, de la bonne sœur d'école maternelle au dominicain polyglotte, autour de 1901. Arrivés en exil, ces spécialistes ont cherché à retrouver les conditions de leur métier, entre pédagogie et vie intellectuelle. C'est à l'enseigne de la langue française que beaucoup ont placé leur survie ou leur reconquête d'un statut. Leur chance a tenu dans le prestige de cette langue : c'est un élément qui distingue les exils de 1685 et de 1901 de ceux d'autres minorités qui n'ont pu espérer emporter dans leurs bagages une langue prestigieuse ou dont l'utilité était internationalement reconnue. Du fond de leur malheur, les bannis de la France royale ou républicaine n'en restaient pas moins 
pourvus de la première langue d'Europe, et pouvaient monnayer ce privilège : l'exilé continuait à parler la langue enviable des maitres du monde ou d'une partie du monde. Tel fut un des premiers paradoxes d'une histoire qui n'en manque pas : dans le Berlin de 1700 comme dans les Beyrouth, Istanbul ou Buenos Aires de 1904, les diasporas françaises apportaient dans leurs cartons le prestige encore intact d'une grande puissance et d'une grande langue.

5 Ainsi cette dernière a-t-elle été disséminée à travers le monde, avec quelques zones de très forte densité, le Levant de nos Atlas 1900, l'Amérique latine «blanche », le Québec, mais aussi, au plus près du territoire métropolitain, les principales villes de la Belgique, de la Hollande, de la Suisse, de l'Espagne, de l'Italie, voire du sud de l'Angleterre. Les poches de francophonie déjà existantes ont été élargies et consolidées, et partout ailleurs le français langue seconde, langue de la distinction, a connu une croissance fulgurante, entrant dans la dot des filles et garçons de beaucoup d'aristocraties et de bourgeoisies, ottomanes, syriennes, égyptiennes, bulgares, roumaines, italiennes, espagnoles, argentines, chiliennes, etc. C'était du reste continuer une tradition ancienne, issue des XVII ${ }^{e}$ et XVIII ${ }^{e}$ siècles, et pour une part liée à l'exil précédent, celui des huguenots. On se trouve là devant l'une de ces ironiques leçons de l'histoire: le malheur d'une partie des Français a fait le bonheur de leur langue, et ce que le pays a perdu en puissance matérielle intérieure (démographique et économique), il l'a regagné en puissance immatérielle extérieure (linguistique et culturelle), même si les deux colonnes ne peuvent évidemment se neutraliser puisqu'il ne s'agit ni des mêmes valeurs ni souvent des mêmes générations. Mais les choses se sont passées comme si la France avait envoyé des dizaines de milliers de cadres chargés d'une seule tâche : bâtir des écoles, recruter des clientèles, diffuser sa langue et ses livres, et cela sans pratiquement verser un seul centime... J'écris : "pratiquement », car la République laïque a versé chaque année au début du $\mathrm{XX}^{\mathrm{e}}$ siècle, au titre du service des Euvres françaises à l'étranger et à la suite d'un débat et d'un vote rituels à la Chambre des députés, une somme oscillant de 600 à 800000 francs et dont chacun savait qu'elle était expressément destinée aux établissements scolaires dirigés par les congrégations religieuses... L'extrême gauche laïque est montée au créneau année après année pour obtenir la suppression de ces crédits, et a trouvé sur son chemin un ministre des Affaires étrangères parlant de l'intérêt supérieur de la nation et réunissant une nette majorité de députés soucieux de financer à Beyrouth des jésuites dont on avait confisqué pour les liquider les bâtiments de métropole... Étonnante France, pétrie de contradictions et de divisions, diront les uns; riche d'un trop-plein de messages et de valeurs, préféreront dire les autres. Je vais suivre ces derniers en montrant la diversité des atouts sur lesquels la France de la Troisième République a pu compter au moment de répandre sa langue dans le monde.

\section{L'accumulation des atouts linguistiques français au début du $X X^{\mathrm{e}}$ siècle}

Ce serait bien réducteur, en effet, que de réduire l'histoire de cette expansion au seul exil des congréganistes. Le jeu français est infiniment plus riche, plus varié, plus fort. Évoquons les cartes maîtresses, rois, reines et as, en quelques mots, au risque d'égrener des banalités, qui n'en sont pas moins justes pour autant. 
7 Il y a tout d'abord l'héritage, le prestige acquis, le français langue de cour et de salon, langue de l'Encyclopédie et de la conversation, seul rival heureux et seul héritier du latin, langue de la république des lettres, celle que des précepteurs de génie ont inculquée aux despotes absolus du XVIII e siècle, celle dont Paul Hazard a montré que la crise de la conscience européenne s'exprimait par son canal, fût-ce au prix de traductions ${ }^{6}$, et dont Rivarol a salué l'universalité. Empruntons à ce méridional qui encore un - termina sa vie en exil, à Berlin, pour être resté fidèle à la monarchie, des formules qui valent pour les années 1900, lorsqu'il parle d'un « empire qu'aucun autre peuple n'a jamais exercé ", celui du théâtre, des habits, du goût, des manières, de la langue, de l'art de vivre, et interroge : «quand on règne par l'opinion, a-t-on besoin d'un autre empire? $\gg^{7}$. Et relisons Hazard, quand il montre que si, dans les années 1680-1715, le leadership intellectuel de l'Europe a basculé « du Midi au Nord» (c'est le titre de l'un de ses chapitres), en clair de la France vers l'Angleterre, c'est précisément grâce à la langue française dans laquelle le grand John Locke est traduit et dans laquelle seule il pouvait alors être lu en Europe ${ }^{8}$. Or, et nous retrouvons le rôle de l'exil huguenot, son traducteur est Pierre Coste, un méridional né à Uzès (à quelques lieues du berceau du futur Rivarol). À terme, souligne Marc Fumaroli', l'universalité de la langue française va se retourner contre la royauté française, puisque les élites françaises peuvent lire sans effort du Locke, le théoricien du contrat social; elles peuvent lire également du Spinoza, traduit par un autre réfugié huguenot, Gabriel de Saint-Glain, ou du Pierre Bayle, cet exilé à Rotterdam qui publie directement en français, sa langue, mais à une époque où l'on peut le faire depuis les Provinces Unies et avec la certitude d'être lu d'un bout à l'autre du continent. Toutes choses égales par ailleurs, le $\mathrm{XX}^{\mathrm{e}}$ siècle a connu un phénomène comparable : c'est en puisant dans la langue et la littérature françaises que les élites des nations colonisées ou dominées par la France allaient se saisir des idées d'émancipation formulées et diffusées par le pays de Montesquieu, de Voltaire, de Michelet ou de Hugo.

Un second atout maître, tout différent, tient au catholicisme. Si le français que parle l'Europe lettrée au XVIII ${ }^{e}$ siècle est plutôt celui du Refuge, de l'Encyclopédie, des Lumières, de Rousseau, éloigné à divers titres de l'Église, il en va différemment au XIX"e, siècle d'or de la reconquête catholique, ouvert dès 1802 par le Génie du christianisme d'un Chateaubriand tout juste rentré de son exil à Londres, et siècle par excellence de toutes les expansions, missionnaire et congréganiste, dans le monde. Quelques centaines de congrégations, fondées pour la plupart à partir des années 1790, recrutent plusieurs dizaines de milliers de religieuses ${ }^{10}$ et religieux qui ne parviennent plus tous à s'employer en France et qui vont fournir des candidats à cette émigration française dont les effectifs sont restés si étiques tout au long du siècle. Le trop-plein de vocations sacerdotales et congréganistes déborde sur Rome, la capitale où il est bon d'entretenir une représentation, surtout si la congrégation est ambitieuse ; sur les pays limitrophes où une clientèle fortunée et distinguée va s'empresser de fréquenter les collègues catholiques français; sur le Québec qui est à la fois haut lieu catholique et haut lieu francophone ; enfin sur les territoires, anciens ou nouveaux, strictement missionnaires ou strictement coloniaux, sur lesquels la France exerce toutes sortes de protectorats, à commencer par le Moyen-Orient ottoman où les « capitulations » signées au XVI ${ }^{e}$ siècle et renouvelées depuis lui ont confié le protectorat des populations chrétiennes autochtones et des missionnaires de tous pays. Plusieurs vagues déposent leurs strates, qui finissent pas constituer des réseaux d'églises, d'écoles, d'œuvres diverses, dont les mailles peuvent être passablement serrées : vieux ordres, comme les capucins, présents 
depuis des siècles en Terre sainte; nouvelles congrégations enseignantes, à l'image des frères des écoles chrétiennes, venues à partir du milieu du XIX ${ }^{e}$ siècle dans une perspective globalement missionnaire; renforts inattendus mais massifs des religieux exilés au début du XX⿳亠口冋.

9 À la veille de la Première Guerre mondiale, les chiffres parlent d'eux-mêmes. En voici de trois sortes: trois missionnaires catholiques sur cinq, dans le monde, sont alors français. Aux États-Unis, en 1901, si les religieuses américaines scolarisent 516100 élèves, les Françaises installées sur place n'en accueillent pas moins de $235202^{11}$. En 1913, le Levant réunit 830 frères des écoles chrétiennes (330 dans le district de Constantinople, 151 dans celui de Jérusalem, 349 dans celui d'Alexandrie), accueillant au total plus de 14000 élèves ; l'Amérique latine, 978 frères et 23664 élèves, le Canada, 748 frères et 22252 élèves, la Belgique, 1281 et 30067 : ces frères sont autant d'agents de la diaspora congréganiste et de l'expansion du français, et il en va de même pour des dizaines d'autres congrégations, surtout féminines. Les voyageurs français en Orient, surtout les voyageurs catholiques... (les laïques militants ne voient pas la même chose, ils s'émeuvent d'une histoire de France qui est celle du jésuite Loriquet, et non de Michelet...), s'émerveillent devant ces classes cosmopolites et pluriconfessionnelles où La Fontaine est récité avec la même émotion que dans les écoles de métropole. Le plus ému, le plus brillant de ces voyageurs est du reste capable de féliciter les écoles juives, dont je vais reparler, à l'égal des écoles congréganistes qu'il est pourtant venu admirer en priorité, afin de contribuer à leur défense devant le Parlement et l'opinion publique : il s'agit de Maurice Barrès en personne, dans Une enquête aux pays du Levant, le récit d'un voyage accompli en mai-juin 1914, mais publié seulement en $1923^{12}$.

La République et le monde laïque ne sont pas en reste. À trois titres. Un quatrième serait trop cynique, il consisterait à se réjouir de la violence anticongréganiste développée par le régime et par contrecoup créatrice pour la langue de cet exil fécond sur lequel je ne reviens pas. Je passe également très vite sur un premier titre, qui ne concerne pas la Méditerranée orientale, sauf à la marge : il tient à la constitution par la République d'un puissant empire colonial dans lequel le français a évidemment ouvert une brillante page de son histoire, quel qu'ait été son lien avec la violence, ici celle de la colonisation. Ces choses-là sont trop évidentes, trop importantes également, elles mériteraient de longs développements que je n'ai pas la place de proposer ici ${ }^{13}$. Faut-il redire que l'Empire a fait du français une langue des mondes arabe et noir, entre autres? Qu'il s'est efforcé de développer son apprentissage, dans les écoles primaires ouvertes aux «indigènes", selon le mot du temps, avec les mêmes ambitions (et les mêmes intolérances négatrices des langues autochtones), les mêmes méthodes, et parfois jusqu'aux mêmes manuels, qu'il faisait au même moment en zone flamande ou au Pays basque ? La très métropolitaine et presque officielle Revue pédagogique résonne de débats, dans les années 1880 et 1890, où l'on voit traiter ensemble de l'Algérie, de la Tunisie, de la Bretagne ${ }^{14}$. Cet aspect des choses me paraît mériter une attention particulière : il prouve que la Bretagne a été la victime d'une même agression d'ordre colonial que la Tunisie - on peut du moins le présenter ainsi. À moins que l'on ne préfère penser que la République a véhiculé du Massif central à la Kabylie le même message, fort à la fois d'une promesse et d'une contrainte : le salut ne pouvait passer que par l'école et la langue françaises. Il y avait sans doute beaucoup à y perdre, du point de vue de l'identité et aussi de la religion, le français de l'école étant alors tout «laïque »; il y avait aussi beaucoup à y gagner, du point de vue de la mobilité et de 
l'entrée dans une communauté linguistique (et peut-être sociale) d'une amplitude considérable.

11 La République, pour revenir à des territoires non coloniaux, ce fut aussi le soutien diplomatique et financier, voire militaire (la démonstration de Mytilène face à l'Empire ottoman en 1901), jamais démenti à l'université Saint-Joseph des jésuites à Beyrouth, par exemple, et à des dizaines d'écoles et de collèges dirigés par les congrégations religieuses. Je me suis risqué dans un autre texte à évoquer le thème d'un "œcuménisme » catholique et laïque dès lors qu'il s'agit d'exporter la langue ${ }^{15}$. Le gouvernement Combes lui-même a pris le soin de mettre cinq grandes congrégations missionnaires françaises à l'abri de sa propre législation anticongréganiste, et il a laissé les ministres des Affaires étrangères, Delcassé, et de l'Instruction publique, Leygues, préparer un amendement autorisant des congrégations enseignantes à garder en France, contre la règle commune, un appareil minimal de recrutement et de formation destiné à assurer le renouvellement des éléments français en poste à l'étranger. Les frères des écoles chrétiennes, pourtant spécialement dans le collimateur du monde laïque, n'ont-ils pas pu entretenir jusqu'en 1909, à Clermont-Ferrand, un "petitnoviciat apostolique ", autorisé dans la seule mesure où il préparait des frères, que l'on initiait à l'espagnol, pour les écoles de l'institut dans l'Amérique latine ? Jusqu'à la veille du Cartel des gauches, les frères ont tenté de mettre en place, avec l'aide de Maurice Barrès dont ce fut le dernier combat ${ }^{16}$, un Institut missionnaire des frères des écoles chrétiennes, car ils étaient persuadés à juste titre que les religieux devaient se présenter désormais comme des agents de la langue française dans le monde pour obtenir au moins l'agrément tacite de la République.

De manière contradictoire, mais que l'on peut juger aussi complémentaire, si l'on regarde les choses à distance, la même République a laissé une partie de ses élites les plus laïques mettre en place, à partir de 1902, une Mission laïque française (MLF), bientôt dotée d'une École normale Jules-Ferry qui n'est autre qu'une École normale pour l'exportation de la pédagogie laïque ${ }^{17}$. Il s'agissait de former une élite de normaliens(nes) du primaire, ou des Écoles de Saint-Cloud et Fontenay, pour leur permettre d'ouvrir écoles ou lycées dans des territoires coloniaux ou que l'on espérait disputer aux congrégations religieuses : la France, laïque à l'intérieur, devait se révéler telle à l'extérieur également. La jeune organisation, parrainée par des ténors de la trempe d'un Doumergue, d'un Herriot, d'un Foncin, mais indépendante d'un gouvernement qui $\mathrm{du}$ reste subventionne bien plus massivement ses rivales congréganistes, ambitionnait rien moins que de se substituer à elles en dépit de l'extrême modestie de ses ressources financières et surtout démographiques. Mais un destin malicieux, toujours supérieurement favorable aux intérêts de la langue française, a voulu que les premiers établissements de la MLF, à Beyrouth et Salonique, deux lieux symboles de la Méditerranée cosmopolite, viennent en complément de ceux des catholiques, en séduisant notamment une clientèle juive. Barrès l'a bien vu, que l'on ne peut pourtant suspecter de sympathie pour la laïcité. Mais il note qu'à Alexandrie un modus vivendi s'est établi entre les jésuites, les frères, l'établissement de l'Alliance israélite universelle et le lycée de la MLF, dirigé par un inspecteur primaire de Paris qui définit son enseignement neutre "avec des phrases de Ferdinand Buisson ». La MLF accueille les chrétiens orthodoxes et les juifs de la classe moyenne, les "grands Juifs » (les grands bourgeois) vont chez les jésuites, les plus modestes à l'école de l'Alliance. Les comptes sont bons : 6600 enfants dans les diverses congrégations françaises, 350 garçons et filles, presque tous juifs, à la Mission laïque, laquelle, « sans nuire aux écoles 
déjà existantes, élargit de quelques mailles le filet que nous jetons sur la jeunesse alexandrine ${ }^{18} »$.

13 L'Alliance israélite universelle, enfin, est de mieux en mieux connue. Elle a été fondée dès 1860 par une poignée de juifs français pour venir en aide aux communautés juives du monde méditerranéen et oriental. Très vite, elle décide de se doter d'un réseau scolaire, et de former les futurs enseignants, recrutés dans les communautés ellesmêmes, dans des écoles normales privées situées aux portes de Paris. La première école primaire ouvre à Tétouan en 1862, les suivantes à Damas et Bagdad en 1864, etc. En 1901, on compte 109 établissements accueillant 29000 élèves; en 1913, 183 et 43700 respectivement ; en 1939, 127 et 47746 encore. La langue de ces écoles est le français, alors que l'usage de l'espagnol chez ces séfarades, ou de l'italien dans sa zone d'influence en Méditerranée orientale ou en Tunisie, eussent été aussi logiques. Outre la fondation et la localisation à Paris de l'Alliance et de ses écoles normales, la raison d'un tel choix tient au prestige persistant, chez les juifs du monde entier, du français comme langue de la liberté et de leur propre émancipation. L'instituteur de Galata, qui n'a nullement la nationalité française, ni ne la recherche, l'exprime magnifiquement, en 1913 : «C'est par la culture française éminemment propice à la diffusion des idées libérales que nous relèverons nos jeunes enfants, déprimés par plusieurs siècles d'oppression et d'abrutissement moral. C'est à un double point de vue la langue par excellence de l'instruction et de l'éducation ${ }^{19}{ }^{\prime}$. Les manuels d'histoire en usage dans les écoles de l'Alliance, rédigés et édités à Istanbul par Moïse Fresco, sont à la gloire de la Révolution française, tout comme ceux qu'ont rédigés un peu auparavant les historiens juifs français. Pour donner une idée de la dévotion libératrice que les juifs de Méditerranée ont nourrie pour la France et sa langue, je ne peux faire mieux que de recourir à Albert Cohen, qui ne fut pas même élève de l'AIU, mais d'une école catholique (on a compris que la clientèle juive se répartissait dans tous les types d'établissements francophones). L'enfant établit "pieusement» sur le rayon d'une armoire fermant à clef une "sainte exposition enfantine ", " une crèche patriotique, une sorte de reliquaire des gloires de la France », dont les reliques sont des portraits de La Fontaine, Corneille, Racine, Molière, Napoléon, Victor Hugo, Lamartine, Pasteur. Le texte s'achève par cette prière :

Ô généreuse et enseignante, France, une de mes patries, et je suis ton vassal et aimant bâtard et fils étranger, car tu m'as fait ce que je suis, car tu m'as nourri du précieux lait de ta mamelle, car tu m'as formé à ton génie, ô souveraine ourdisseuse des mots, ô discernante, car tu m'as donné ta langue, haut fleuron de l'humaine couronne, ta langue qui est mienne et pays de mon âme, ta langue qui m'est aussi une patrie ${ }^{20}$.

\section{L'élection, l'annonce, l'inquiétude}

14 Catholique, laïque, juive... Qu'est-ce qu'est exactement la France dans les années 1880-1930, surtout lorsqu'elle propose sa littérature et sa langue aux clientèles méditerranéennes? Un peu de tout cela? Est-elle pour le moins double, au mauvais sens du mot, celui de duplicité ? Voire triple? Un observateur suisse, Paul Seippel, publie un livre intitulé Les deux Frances ${ }^{21}$; quelques années plus tard, Péguy vante les «deux vocations » du pays. Leurs deux n'ont pas le même sens, l'un est exclusif, l'autre inclusif. J'ai déjà trop souvent cité la phrase de Péguy qui suit, un lecteur attentif a le droit de s'en agacer, mais cette phrase est simplement une des plus belles et des plus 
profondes qui aient été écrites sur mille ans d'histoire de la France, peut-on se priver de la donner à lire une fois encore ? Et Péguy n'est-il pas à lui seul la plus forte icône de cette époque miraculeuse où la langue française est à la fois catholique, laïque (la langue de ses chers hussards noirs), socialiste, hébraïque, et déjà méditerranéenne, africaine, canadienne, antillaise... ? Voici, donc, ces quelques lignes de L'Argent, suite, en 1913 :

Il ne fait aucun doute que la France a deux vocations dans le monde. [...] La France n'est pas seulement la fille aînée de l'Église, (et ceci apparaît constamment et avec une fidélité surprenante); elle a aussi dans le laïque une sorte de vocation parallèle singulière, elle est indéniablement une sorte de patronne et de témoin, (et souvent une martyre), de la liberté dans le monde. [...] Telle est notre double charge. Telle est notre double garde ${ }^{22}$.

15 C'est de ce côté cumulatif et complémentaire que l'historien de la langue française doit regarder. J'ai assez dit que la France additionne en Méditerranée orientale, avant même les mandats de la SDN, plusieurs sortes de réseaux, d'instituteurs, de maisons d'école, de locuteurs. Le jeu qu'elle a en mains est riche de cartes de toutes les couleurs, rouges et noires, pique et cœur. Et une même trame circule de l'une à l'autre, qui renvoie à une très vieille définition que la France se donne d'elle-même: c'est une trame " évangélique », une annonciation aux peuples qu'une bonne nouvelle est advenue à Paris, qui les intéresse tous et qui touche à la liberté - celle de la terre sainte au temps des croisades, peut-être, ou celle des chrétiens d'Orient depuis l'intervention de François $\mathrm{I}^{\text {er }}$; et surtout, celle de l'humanité tout entière, juifs compris, au temps de 1789, un message réapproprié et réactualisé par une Troisième République qui sait en être l'héritière directe et le bras, armé ou pédagogique, dans le monde. Rarement nom fut mieux et plus audacieusement trouvé que celui de la Mission laïque française: laïque, certes, et française, mais surtout missionnaire. Est-elle rien d'autre, par ailleurs, cette Alliance israélite universelle, qui depuis Paris prétend porter aide et instruction, au nom des valeurs révolutionnaires et républicaines françaises, aux juifs du monde entier ? Et cette Alliance française, consœur des précédentes en matière de diffusion du français mais aussi de conception de l'humanité ? Certes, on peut multiplier les distinctions, insister sur les oppositions farouches qui dressent la Mission laïque contre les congrégations, ou ces dernières contre la première et contre les juifs qui la soutiennent, ou contre la République laïque qui soutient une association juive... Mais les frères ennemis eux-mêmes l'avaient perçu, la page de Barrès citée plus haut le montre bien, ces oppositions ne se défont pas mutuellement, elles font l'inverse, chacune tissant sa part à elle, sa pièce, plus ou moins grande, de ce qui ressemble à l'arrivée à ce magnifique manteau d'arlequin, parfois troué ou taché, que l'on devait appeler plus tard la francophonie. Si le carton de cette tapisserie a trop souvent été dessiné par la seule colonisation, l'exemple de la Méditerranée orientale révèle d'autres responsabilités et d'autres héritages, certes chargés de confrontation, de conflit, d'exportation de modèles venus d'Europe (ou revenus, si l'on songe au christianisme), mais indépendants de la colonisation et renvoyant la France et sa langue à une histoire plus longue, plus complexe et plus riche.

16 Faut-il terminer sur un tel satisfecit, une telle réconciliation franco-française, un siècle seulement après ces lois par lesquelles les uns contraignirent les autres à l'exil, beaucoup des seconds s'étant par ailleurs déconsidérés dans leurs haines antidreyfusarde et antisémite ? Plusieurs textes du début du $\mathrm{XX}^{\mathrm{e}}$ siècle trahissent déjà (mais pouvaient-ils oublier, en amont, l'étrange défaite de 1870?) une inquiétude 
majeure qui n'est peut-être, somme toute, que l'avers de la fierté missionnaire et du sentiment d'une élection: la peur du déclin généralisé, y compris dans le domaine linguistique qui est pourtant celui dans lequel la France engrange quelques-uns de ses plus beaux résultats. Des coïncidences thématiques et chronologiques retiennent l'attention, à cet égard. C'est en 1903 que Georges Goyau, le futur historien des missions, publie un livre en diptyque, à propos des luttes d'influence au Levant : Vieille France. Jeune Allemagne $e^{23}$; les épithètes disent à peu près tout : même si la prééminence va à l'ancienneté d'une présence, le dynamisme conquérant, voire agressif, joue au profit de la jeune rivale... La même année, un sociologue d'origine russe, Jacques Novicow, publie L'expansion de la nationalité française ${ }^{24}$, entendons de la langue française. La Revue des Deux Mondes a rendu compte au moins deux fois de cet essai. Anatole LeroyBeaulieu adhère à ses thèses et prend l'exemple du Levant pour appeler à l'union de tous les réseaux scolaires français qui agissent sur place ${ }^{25}$; cette position ne surprend pas chez un homme qui avait été à la fois catholique et dreyfusard et venait d'écrire un petit livre iconoclaste, Les Doctrines de haine, pour renvoyer dos à dos les antisémites et les anticléricaux. Mais la supplique en faveur d'une union sacrée des diffuseurs du français est le signal d'une inquiétude grandissante face à la multiplicité des concurrences extérieures. Ferdinand Brunetière est beaucoup plus pessimiste : il prend l'exemple de la langue grecque, qui a certes conquis le monde hellénistique et romain. " Mais la Grèce, où était-elle ? " ${ }^{26}$. Comprendre : « Mais la France, où est-elle? ", même si sa langue est au centre de la nouvelle koinè hellénistique des années 1900. Au temps de la guerre froide ou de la mondialisation, la question serait peut-être devenue : « La France, combien de divisions dans le monde ? Combien de parts de marché ? ». Un assez bon nombre, répondrait-on volontiers, du point de vue linguistique, quoique tout immatérielles, quoique liées à des souvenirs scolaires, à des livres, des récitations, des rédactions. Mais aussi des lectures continuées à l'âge adulte, une presse qui forme le regard, des conversations qui contribuent à définir une identité et une appartenance, voire des modes de consommation, ou encore, en cas de crise, une direction potentielle d'émigration (on songe aux juifs du Maghreb, aux chrétiens du Liban, peut-être aux Arméniens).

17 C'est une chose bien délicate que de mesurer le poids diplomatique, économique, politique d'une langue. Mais c'est une chose nécessaire, qu'il ne faut ni surévaluer ni traiter à la légère. Il suffit, du reste, d'observer le privilège mondial de l'anglais, aujourd'hui, pour mieux apprécier la chance dont le français, et ceux dont il était la langue maternelle ou seconde, ont pu jouir il y a quelques dizaines d'années. Une " chance " surgie de multiples sources, y compris les plus politiquement immorales, avec l'exil de vaincus. Une chance pluraliste, multiconfessionnelle, judéo-catholique et catholico-laïque (osons ces traits d'union qui n'étaient que des fronts de conflit), et qui a légué à la langue française actuelle quelques très beaux territoires et très grands écrivains et peut léguer à la France actuelle un beau sujet de méditation sur ce qu'apportent à un pays non pas le rêve d'unité mais la couture même maladroite de ses polyphonies et de ses accents. 


\section{NOTES}

1. Cl. Hagège, Le Souffle de la langue. Voies et destins des parlers d'Europe, Odile Jacob, "Opus », 1994 [1992], p. 80-83.

2. Ce texte est largement une synthèse de publications précédentes dont je crois honnête de donner les références : "Les deux vocations : catholique ou laïque ? La France, le Levant et l'Islam, 1860-1920», Religion et identité, sous la direction de Gabriel Audisio, Aix-en-Provence, Publications de l'université de Provence, 1998, p. 199-209; « Langue et diasporas. Trois exemples d'expansion paradoxale du français », Diasporas. Histoire et sociétés, $n^{\circ} 2,2003$, p. 102-119; et l'introduction à Une France en Méditerranée, dont la référence est donnée en note 12 et aux vingt-cinq articles auxquels je prends la liberté de renvoyer tout spécialement.

3. Spécialement l'exil républicain à la suite du coup d'État du 2 décembre 1851; les congréganistes, jésuites en tête, ont connu un premier exil en 1880, à la suite des décrets signés par Jules Ferry, mais ce ne fut qu'une répétition du grand exil qui allait suivre.

4. Au contraire, même, dans le cas des protestants, puisqu'il leur était interdit de quitter le Royaume après 1685 , sous peine de la prison ou des galères à vie.

5. Ce «mythe» de la faute de Louis XIV contre la France a été étudié par Myriam Yardeni, « Naissance et essor d'un mythe : la révocation de l'édit de Nantes et le déclin économique de la France ", in idem, Repenser l'histoire : aspects de l'historiographie huguenote des Guerres de religion à la Révolution française, Champion, 2000, p. 191-206.

6. Voir les deux ouvrages édités par Jens Häseler et Antony McKenna, La Vie intellectuelle aux Refuges protestants (le tome II a pour sous-titre Huguenots traducteurs), Champion, 1999 et 2002. 7. Cité par Marc Fumaroli, «Le génie de la langue française », Pierre Nora, dir., Les lieux de mémoire, III, Les France. 3. De l'archive à l'emblème, Gallimard, 1992, p. 967.

8. Paul Hazard, La Crise de la conscience européenne 1680-1715, Fayard, 1961 [1933].

9. M. Fumaroli, op. cit., p. 963-964. Du même auteur, Quand l'Europe parlait français, Éditions de Fallois, 2001.

10. Cf. Claude Langlois, Le catholicisme au féminin, Cerf, 1984.

11. Note de l'ambassadeur Jules Cambon du 12 juin 1901, résumée dans une Note pour le directeur des Affaires politiques, 17 novembre 1904, Archives du ministère des Affaires étrangères, Saint Siège NS 23, fo 249.

12. Dans la Revue des Deux Mondes, du 15 février au $1^{\mathrm{er}}$ novembre, puis en deux volumes chez Plon-Nourrit. Réédition en un volume aux Éditions Manucius, Houilles, 2005. J'ai analysé et cité cet ouvrage dans «Trois France en Méditerranée orientale : l'empire immatériel de la langue », introduction à $\mathrm{P}$. Cabanel, dir., Une France en Méditerranée. Écoles, langue et culture françaises $\mathrm{XIX}^{\mathrm{e}}-\mathrm{XX}^{\mathrm{e}}$ siècles, Créaphis, 2006, p. 9-29.

13. Voir la notice «Langue française et francophonie » dans Claude Liauzu, dir., Dictionnaire de la colonisation française, Larousse, 2007, p. 397-399.

14. Voir notamment Jean-François Chanet, L'école républicaine et les petites patries, Aubier, 1996 ; P. Cabanel, «Catholicisme et laïcité, langue et nation à la fin du XIX siècle, Félix Pécaut et les Basques ", Jean Jaurès, Cahiers trimestriels, n 152, avril-juin 1999, Les “ Petites patries " dans la France républicaine, p. 77-90 ; «Suisse romande, Méditerranée séfarade, Tunisie coloniale... et Bretagne: frontières et complexité dans l'apprentissage du français républicain », L'école française et les langues régionales, colloque de Montpellier, 13-14 octobre 2006, Publ. de l'université Paul-Valéry Montpellier 3, coll. «Études occitanes », 2007.

15. Voir la référence donnée en note 12.

16. Cf. son ouvrage posthume, M. Barrès, Faut-il autoriser les congrégations ?, Plon-Nourrit, 1924. 
17. André Thévenin, La Mission laïque française à travers son histoire 1902-2002, Mission laïque française, 2002 ; Dialogues. Bulletin de liaison des professeurs français à l'étranger, «Éléments pour une histoire de la Mission laïque française 1902-1982 ", n 35-36-37, 1982.

18. M. Barrès, Une enquête..., op. cit., éd. 2005, p. 30.

19. A. Benveniste, cité par Aron Rodrigue, De l'instruction à l'émancipation. Les enseignants de l'Alliance israélite universelle et les Juifs d'Orient 1860-1939, Calmann-Lévy, 1989, p. 117. Galerie de portraits d'enseignants de l'Alliance dans Élisabeth Antebi, Les Missionnaires juifs de la France 1860-1939, Calmann-Lévy, 1999.

20. A. Cohen, Ô vous, frères humains, in đeuvres, Gallimard, « Bibliothèque de la Pléiade », 1993, p. 1065.

21. P. Seippel, Les Deux Frances, Lausanne, Payot, Paris, Alcan, 1905.

22. Ch. Péguy, L'Argent, suite, Cuures en prose complètes, Gallimard, "Bibliothèque de la Pléiade ", III, 1992, p. 948.

23. G. Goyau, Les nations apôtres. Vieille France, jeune Allemagne, Perrin, 1903.

24. Colin, 1903. L'auteur revient sur ces questions dans un article paru dans la Revue des Deux Mondes, «La langue auxiliaire du groupe de civilisation européen. Les chances du français », $1^{\text {er }}$ décembre 1907, p. 553-588.

25. A. Leroy-Beaulieu, «La langue française et les révolutions de l'Orient », Revue des Deux Mondes, 15 avril 1909, p. 832-871.

26. F. Brunetière, «Une apologie de la langue française », Revue des Deux Mondes, 1903, p. 597.

\section{RÉSUMÉS}

Une des formes d'expansion de la langue française repose sur un paradoxe, pour ne pas dire une «faute morale » : ce sont des exilés qui l'ont assurée en partie, les huguenots à partir du XVII siècle, les congréganistes au début $\mathrm{du} \mathrm{XX}^{\mathrm{e}}$. Il y a un second paradoxe : la République laïque a assumé l'héritage catholique et missionnaire du pays, achevant ainsi de donner à sa langue une extraordinaire série d'atouts. Plusieurs réseaux la véhiculent dans le monde : les missionnaires, les congréganistes en exil, les instituteurs de l'Empire colonial, ceux de la Mission laïque créée en 1902, et ceux (et celles) enfin de l'Alliance israélite universelle (depuis 1860). Ces réseaux sont parfois hostiles les uns aux autres, mais leurs efforts complémentaires ont donné à une France à la fois sûre de son destin mondial, et inquiète face à un déclin toujours annoncé, une autre forme de puissance, immatérielle mais précieuse.

It is on a paradox, not to say a " moral error » that French managed to expand. First, it found its way through thanks to the exiled, first the Huguenots in the 17th century and later the Congregationists in the beginning of the 20th century. Secondly, the lay Republic endorsed the Catholic and missionary heritage of the country, contributing thus to the number of assets given to its language. French has been promoted through several networks - missionaries, exiled Congregationists, the colonial empire teachers, but also teachers sent by the Mission laïque created in 1902 and by the Alliance israélite universelle since 1860. These networks have shown hostility towards each other sometimes. However, they gave France, which was at the same time worried of declining but sure of its global destiny, a certain form of immaterial but precious power. 
INDEX

Keywords : French language, expansion, spreading, networks, global destiny, decline

Mots-clés : langue française, expansion, diffusion, réseaux, destin mondial, déclin

\section{AUTEUR}

\section{PATRICK CABANEL}

Université Toulouse-Le Mirail, France 